VII Congresso Brasileiro de Informática na Educação (CBIE 2018)

Anais do XXIX Simpósio Brasileiro de Informática na Educação (SBIE 2018)

\title{
Socialsearch: uma ferramenta de apoio pedagógico para mediação da aprendizagem nas redes sociais digitais
}

\author{
Carlos Emilio Padilla Severo ${ }^{1}$, Rodrigo Figueira de Freitas ${ }^{1}$ \\ ${ }^{1}$ Departamento de Ensino, Pesquisa e Extensão - Instituto Federal Sul-rio-grandense \\ (IFSul) - Campus Bagé - RS - Brasil \\ carlossevero@ifsul.edu.br,foolha@gmail.com

\begin{abstract}
The text presents the results of a pilot study using a technology to map the textual interactions between teacher and students in a group on the social network Facebook. The purpose of the technology was to help the teacher, who uses online environments as pedagogical resources, in the teaching and learning process. The results of the pilot study allowed a reflection on the potentialities and limitations of technologies as resources to support education in the face-to-face modality, aiming at expanding the spacetime of teaching pedagogical practice.
\end{abstract}

Keywords: technologies in education, pedagogical mediation, virtual environments, online social networks, mapping of interactions.

Resumo. O texto apresenta os resultados de um estudo piloto a partir da utilização de uma tecnologia para mapeamento das interações textuais entre professor e estudantes em um grupo na rede social Facebook. A tecnologia teve como propósito o auxílio ao docente, que utiliza ambientes online como recursos pedagógicos, no processo de ensino e aprendizagem. Os resultados do estudo piloto permitiram uma reflexão acerca das potencialidades e limitações das tecnologias como recursos de apoio a educação na modalidade presencial, visando a ampliação do espaço-tempo na prática pedagógica docente.

Palavras-chave: tecnologias na educação, mediação pedagógica, ambientes virtuais, redes sociais online, mapeamento de interações.

\section{Introdução}

A tecnologia das redes sociais online está presente no cotidiano das pessoas na sociedade atual. A evolução dos meios de comunicação, proporcionados pela Internet, permitem uma maior aproximação dos membros da sociedade. Com isso, o acesso à informação e troca de conhecimento, proporcionados pelos ambientes das redes sociais online, apresentam possibilidades para ampliação de estratégias para o desenvolvimento da aprendizagem.

Trata-se de um ambiente fértil para propostas educativas que visem a construção de conhecimento a partir da colaboração entre os membros de uma comunidade, onde o aprendizado possa ocorrer pela reflexão dos indivíduos por meio de conversas, trocas de experiências, resolução de problemas, elaboração de projetos e demais atividades através do trabalho mútuo e participativo.

Segundo Panitz (1998), a colaboração não é somente uma estratégia de 
VII Congresso Brasileiro de Informática na Educação (CBIE 2018)

Anais do XXIX Simpósio Brasileiro de Informática na Educação (SBIE 2018)

planejamento das atividades pedagógicas, também pode ser entendida como uma filosofia de ensino do educador. Na aprendizagem pautada na colaboração deve haver respeito entre os pares, onde as habilidades individuais devem ser valorizadas no intuito de contribuição com o trabalho do grupo. Não há indivíduo melhor que o outro, tal premissa deve ser aplicada em todas as interações entre os indivíduos que compõem uma comunidade. Harassim (1989), complementa a ideia de colaboração, informando que em um processo educativo colaborativo os indivíduos trabalham para criação de significado, onde a exploração de um tema de aprendizagem visa o desenvolvimento de habilidades cognitivas entre os membros de um grupo. De acordo com Cord (2000), a Internet é uma ferramenta contemporânea adequada a implantação de práticas pedagógicas colaborativas, visto que permite a constante interação entre as pessoas, possibilitando que as trocas levem à concepção do produto final que é a construção da aprendizagem.

Neste sentido, evidencia-se a importância da Internet, mais especificamente das redes sociais online, para o desenvolvimento da aprendizagem e habilidades cognitivas de um grupo de estudantes. Além disso, destaca-se a importância do trabalho colaborativo como estratégia pedagógica para o desenvolvimento da aprendizagem individual do estudante. Entretanto, o ambiente das redes sociais online não apresenta uma estrutura que motive o docente durante suas atividades de mediação pedagógica, visto que as postagens seguem uma organização linear, de acordo com a ordem cronológica das interações entre os indivíduos, o que instigou algumas questões de pesquisa: como a tecnologia pode auxiliar o docente na identificação de estudantes com maior necessidade de mediação pedagógica? De que forma as interações de um grupo de estudantes podem ser organizadas e mensuradas? Como o docente pode fazer uso das redes sociais como ambiente para o desenvolvimento de uma pedagogia colaborativa?

Sendo assim, neste trabalho é apresentada uma tecnologia que extrai e organiza as interações textuais de um grupo de estudantes da rede social Facebook, apresentando ao docente uma interface gráfica, onde podem ser observadas e analisadas as interações entre os indivíduos, cujo objetivo foi verificar como o ambiente online pode ser um espaço complementar para o desenvolvimento da aprendizagem do estudante. Assim, ampliando-se os espaços de interação entre professor-estudante e estudante-estudante, buscando-se contornar as limitações da sala de aula física tradicional em relação ao tempo e espaço de aprendizagem. Para validação da tecnologia, foi realizado um estudo piloto, aplicado a um grupo de estudantes de um curso de Tecnologia em Análise e Desenvolvimento de Sistemas, o que levou a reflexões e questionamentos acerca da utilização de tecnologias digitais como recursos pedagógicos no processo de constituição da subjetividade e influência na apropriação do conhecimento pelo estudante.

Para fundamentação da proposta investigativa foram levantados conceitos acerca da influência das redes sociais na educação, bem como, aspectos sobre a mediação pedagógica nos ambientes online. Na sequência, a abordagem metodológica do trabalho é apresentada, seguida de uma análise e discussão dos resultados do estudo piloto. Algumas considerações finais são expostas a título de fechamento das ideias apresentadas ao longo do texto.

\section{Fundamentação teórica}

De acordo com Wellman (1997), rede social é uma estrutura que interliga pessoas, ou 
VII Congresso Brasileiro de Informática na Educação (CBIE 2018)

Anais do XXIX Simpósio Brasileiro de Informática na Educação (SBIE 2018)

grupos de pessoas, por meio das interações ou laços sociais comuns. A comunicação na rede social é realizada através de uma linguagem com uma notação própria, a qual está relacionada à cultura dos indivíduos que a constituem (CAPRA, 2008). Tais redes vêm influenciando as relações nas mais distintas atividades humanas, tais como: economia, cultura e educação.

Juliani et al. (2012), relata que as redes sociais online tiveram uma grande evolução desde os seus desenvolvimentos, passando por um grande processo de aperfeiçoamento e modificações. O que antes era apenas uma oferta de comunicação via rede de dados, através de ferramentas para troca de mensagens entre usuários, tornou-se rede social a partir do surgimento dos primeiros sites. Uma das primeiras redes sociais online foi a Orkut, que após ser adquirida pela Google já não existe mais. Atualmente, a Facebook se tornou a rede social online com a maior quantidade de usuários no Brasil.

Neste trabalho, explora-se o espaço virtual das redes sociais online na constituição de comunidades de aprendizagem. Wellman (1997) postula que as comunidades, sejam virtuais ou não, são redes que geram relações de identidade entre os indivíduos, onde o sentido de pertencimento e integração é a tônica que mantém a socialização e constituição de uma identidade. $O$ foco de interesse foi investigar e refletir acerca do potencial das redes sociais virtuais no contexto de comunidades de aprendizagem. De acordo com Moran (2015), o interesse nas comunidades de aprendizagem virtuais tem crescido, devido a fatores como: a relevância do conhecimento nas sociedades contemporâneas, bem como, a constante inovação tecnológica e sua incorporação aos hábitos dos indivíduos. Além disso, cabe ressaltar as diversas possibilidades de colaboração no processo de desenvolvimento da aprendizagem do estudante, a partir das trocas de experiências, conhecimentos e auxílio mútuo.

Entretanto, Filatro e Piconez (2013), salientam que não basta inovações em tecnologias e conteúdos digitais disponíveis nos ambientes online para o processo educativo, mas que ocorra a constante interação entre pessoas, bem como, entre pessoas e os conteúdos digitais disponíveis pelas ferramentas. Para Andrade e Vicari (2011), a interação entre os indivíduos de um grupo é essencial e ocorre por meio de ações recíprocas entre os membros de uma coletividade, as quais visam um determinado fim. As autoras complementam, destacando que a interação entre os indivíduos está inserida em um processo de mediação, o qual ocorre através nas diversas ferramentas disponíveis no ambiente virtual.

Este trabalho visou a exploração do potencial educativo das redes sociais online. Principalmente, no que diz respeito a um ambiente de ampliação de oportunidades de ensino e aprendizagem, expandindo o espaço e tempo de mediação pedagógica docente, não limitando-se somente ao espaço e tempo proporcionados pela aula presencial.

Em pesquisas recentes é evidenciada a preocupação e importância acerca do controle da própria aprendizagem por parte do estudante (GUEDES-GRANZOTTI et al., 2015; MORAN, 2015; PAIVA et al., 2016; SILVA et al., 2014). A prática de ensino docente deve estar centrada no estudante, substituindo-se as tradicionais aulas expositivas pela aprendizagem que contemple o desenvolvimento da autonomia do estudante. As estratégias de ensino não devem ser pautadas em um perfil de estudante de anos atrás, deve-se proporcionar condições que contemplem os hábitos dos nativos digitais - termo cunhado por Prensky (2009). De acordo com o autor, o nativo digital 
VII Congresso Brasileiro de Informática na Educação (CBIE 2018)

Anais do XXIX Simpósio Brasileiro de Informática na Educação (SBIE 2018)

pensa e processa a informação de forma diferente das gerações passadas, pois já está integrado com os recursos disponíveis pelas tecnologias digitais.

Dessa forma, não devemos negligenciar as tecnologias propostas pelas redes sociais online, visto que este é um dos canais de interação preferidos do nativo digital. Os ambientes virtuais das redes sociais possibilitam o diálogo, colaboração, construção coletiva de conteúdo e reflexão. A utilização apropriada das ferramentas de interação disponíveis nas redes sociais online pode promover a autonomia do estudante.

Moore (2007), esclarece que a distância entre estudante e professor nos ambientes virtuais não é geográfica, mas sim, pedagógica. O rompimento dessa distância pedagógica ocorre pelo delineamento da natureza das interações no ambiente virtual (distância transacional). Aliado a isso, temos que levar em consideração a forma como o estudante aprende. Para Prensky (2009), não podemos planejar nossas práticas pedagógicas sem entender o perfil do nativo digital. De acordo com o autor o estudante atual está imerso em tecnologias, fato que altera a forma como desenvolve sua aprendizagem. A constituição da subjetividade do estudante é influenciada pelas constantes interações e carga de informações que recebe no dia a dia.

Moore (2007) explica que o aprendizado apoiado por ambientes virtuais deve estar alicerçado em estratégias que levem em consideração os aspectos inerentes da tecnologia. O que difere do modelo de planejamento de aula presencial. Além disso, a mediação online pode ser considerada como acompanhamento, orientação e articulação instigadora, as quais são tarefas realizadas pelo professor, com base nas trocas e construção de conhecimento entre os estudantes por meio das ferramentas digitais (RANGEL et al., 2008).

Dada a importância da mediação no desenvolvimento da aprendizagem do estudante, este trabalho descreve os resultados do processo de construção de uma tecnologia para auxílio ao docente na extração de informações acerca das interações de natureza pedagógica em ambientes online. Para isso, foi aplicado um estudo piloto na rede social Facebook, cujo objetivo foi verificar como pode ser um espaço complementar para o desenvolvimento da aprendizagem do estudante. Assim, ampliando-se os espaços de interação pedagógica, buscando-se contornar as limitações da sala de aula física tradicional em relação ao tempo e espaço.

Os resultados do estudo de piloto levaram a reflexões e questionamentos acerca da utilização de tecnologias digitais como recursos pedagógicos no processo de constituição da subjetividade e influência na apropriação do conhecimento por parte do estudante.

\section{Abordagem metodológica}

O trabalho foi de natureza qualitativa e exploratória, onde foi construída uma ferramenta intitulada Socialsearch, para auxiliar o docente no mapeamento das interações entre professor e estudante, bem como, entre os próprios estudantes em grupos da rede social Facebook. A ferramenta extrai as postagens textuais, cujo objetivo é apresentar graficamente ao docente as contribuições dos participantes durante o desenvolvimento das atividades pedagógicas. Dessa forma, permitindo o acompanhamento das interações e desenvolvimento da aprendizagem dos estudantes com apoio do ambiente virtual. Trata-se de uma proposta, com base tecnológica, visando a constituição de um espaço extraclasse para discussões acerca dos conceitos 
VII Congresso Brasileiro de Informática na Educação (CBIE 2018)

Anais do XXIX Simpósio Brasileiro de Informática na Educação (SBIE 2018)

\section{5}

abordados em uma disciplina, ampliando o espaço de aprendizagem dos estudantes para além da sala de aula física tradicional.

As seguintes etapas constituíram a abordagem metodológica do trabalho:

- estudo da base teórica, onde foram levantados e selecionados os referenciais teóricos para fundamentação de aspectos sobre redes sociais na educação, os ambientes virtuais como espaço de aprendizagem, ferramentas digitais na educação e a mediação em ambientes online;

- levantamento de requisitos, nesta etapa foram definidos os requisitos da aplicação, delimitando-se as funcionalidades desejadas e mais importantes para o processo de mapeamento das interações;

- definição de tecnologias, onde foi realizado o estudo da base tecnológica envolvida na construção do mecanismo de coleta e organização das postagens textuais realizadas em um grupo do Facebook;

- prototipação do mecanismo tecnológico, a qual envolveu a elaboração do software que utilizou a API (Application Programming Interface) do Facebook para buscar as postagens textuais e, posteriormente, organização das postagens para mapeamento das mediações ocorridas.

- aplicação de um estudo piloto, realizado para avaliar e testar as funcionalidades do mecanismo tecnológico. Para isso, um grupo de estudo da disciplina de Programação Orientada a Objetos foi criado no Facebook. O estudo piloto foi realizado de agosto a novembro de 2017, o grupo foi constituído por 12 estudantes.

- análise e discussão dos resultados a partir dos dados coletados pela ferramenta para a verificação e organização das interações pedagógicas no ambiente online.

Como instrumento de coleta de dados foi realizada a observação participativa do pesquisador, visando-se explorar o ambiente virtual do Facebook como recurso pedagógico, além de verificar o comportamento da ferramenta Socialsearch durante as atividades de mapeamento das interações e renderização dos resultados. Com isso, visando-se descrever e interpretar o processo de desenvolvimento da aprendizagem dos estudantes para identificação de necessidades de mediação pedagógica entre os componentes do grupo.

A próxima seção aborda aspectos relacionados a construção da Socialsearch e suas funcionalidades em relação ao mapeamento de interações entre os participantes de um grupo de estudantes no Facebook.

\section{Materiais e métodos}

A ferramenta foi construída em camadas, visando-se a separação de responsabilidades do software. Em cada camada foi utilizada alguma tecnologia, cuja integração constitui o software como um todo. O conjunto de todas essas camadas formam a SocialSearch. A seguir, será descrito o modelo conceitual da aplicação, o qual apresenta as relações entre as principais entidades de dados da aplicação, entre elas:

- grupos: os quais representam o conjunto de estudantes da disciplina e onde ocorrerão as interações pedagógicas;

- posts: representam as interações textuais dos estudantes e professor no grupo do Facebook. A partir das contribuições na forma de posts é que serão 
VII Congresso Brasileiro de Informática na Educação (CBIE 2018)

Anais do XXIX Simpósio Brasileiro de Informática na Educação (SBIE 2018)

6

mapeadas as interações pedagógicas entre os participantes do grupo;

- usuários: esta classe representa o modelo de dados dos estudantes e professor que participam de um grupo de interação da disciplina;

- comentários: a partir dos posts dos estudantes e professor da disciplina, também, podem ser inseridos comentários acerca de contribuições e dúvidas postadas. Dessa forma, pode-se analisar as interações e a realização do levantamento de dificuldades encontradas pelos estudantes ao longo da disciplina.

Um grupo é constituído pelas postagens realizadas pelos usuários (estudantes e professor), bem como, cada postagem pode ser complementada por comentários dos participantes. Sendo assim, em um grupo pode ser mantida uma lista de postagens realizadas, visando-se o mapeamento dos usuários que interagem com o grupo. Os comentários associados a uma postagem são de suma importância para o mapeamento das interações, pois, a partir da identificação de comentários, pode-se realizar o mapeamento de mediações realizadas entre professor e estudante, ou entre os próprios estudantes.

A Figura 1, ilustra o painel de controle contendo informações básicas do grupo, tais como: número de usuários, número de postagens, comentários, data da última coleta de dados no Facebook e o botão para realização de uma nova coleta. Logo abaixo, uma linha do tempo formada pelas postagens do grupo é apresentada na interface gráfica da ferramenta. Cada participante do grupo é apresentado com sua foto de perfil, bem como, o conteúdo das postagens realizadas. As imagens e informações de identificação estão desfocadas para fins de preservação da identidade dos estudantes.

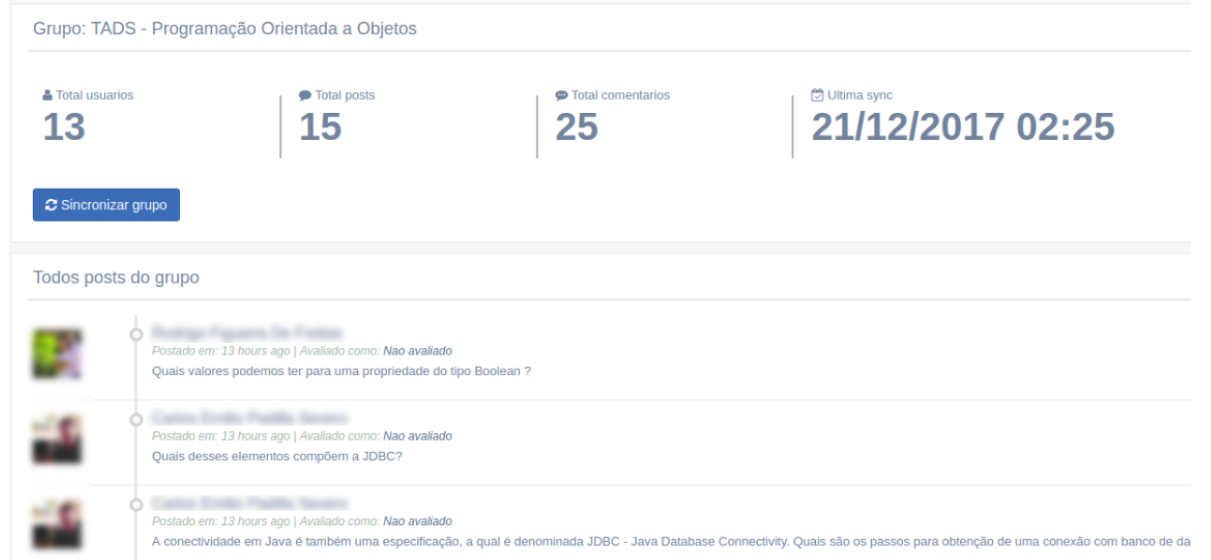

Figura 1. Visão geral de parte do painel de informações do grupo. Fonte: própria autoria

Algumas estatísticas são apresentadas ao docente da disciplina a partir do mapeamento dos dados coletados. Portanto, para cada usuário do grupo é elaborado um cartão contendo as seguintes informações: a quantidade de postagens realizadas por estudante, comentários realizados pelos participantes e o percentual de contribuição individual, conforme ilustrado na Figura 2. 
VII Congresso Brasileiro de Informática na Educação (CBIE 2018)

Anais do XXIX Simpósio Brasileiro de Informática na Educação (SBIE 2018)

7

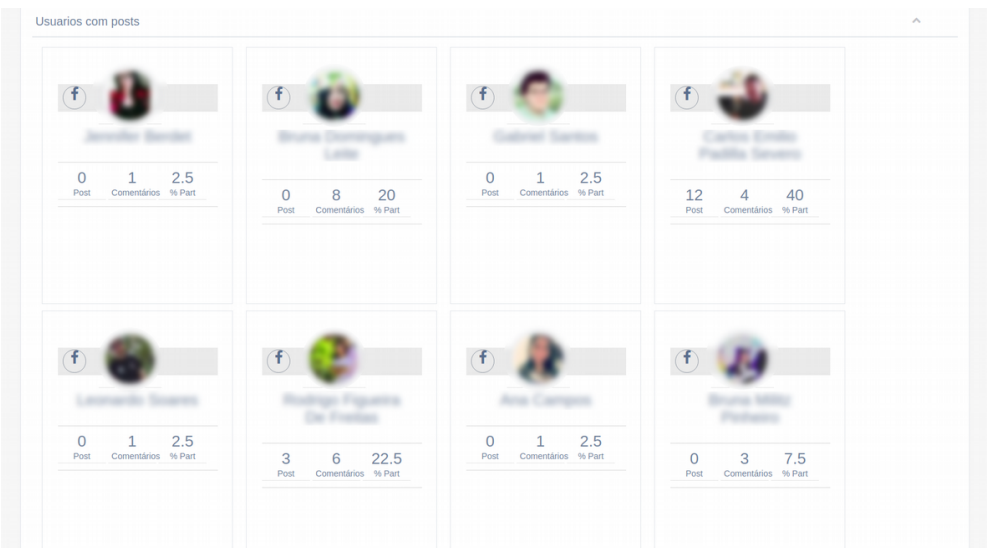

Figura 2. Conjunto de cartões de participantes no grupo. Fonte: própria autoria

Outra funcionalidade da ferramenta é a possibilidade de o docente regente poder verificar quais estudantes não estão interagindo no grupo da disciplina. Tal aspecto é relevante, pois a falta de interação pode estar relacionada a dificuldades de aprendizagem do estudante. Para isso, é apresentado outro painel com a identificação de cada estudante com sua foto e outras informações. Na sequência do texto, uma análise e reflexão acerca dos resultados apontados no estudo piloto, utilizando-se a ferramenta para visualização das interações pedagógicas realizadas no grupo do Facebook, são apresentadas.

\section{Análise e discussão dos resultados}

Neste trabalho, pode-se observar a utilidade do Facebook como uma ferramenta pedagógica para ampliação do espaço de ensino e aprendizagem de estudantes, permitindo a discussão e reflexão de temas abordados em uma disciplina, através de postagens e comentários realizados pelos participantes de um grupo.

As interações entre os próprios estudantes, visando o desenvolvimento da aprendizagem, de forma colaborativa, puderam ser constadas durante a aplicação do estudo piloto. $\mathrm{O}$ trabalho investigativo permitiu a observação do desenvolvimento da capacidade de reflexão e argumentação do estudante ao longo do curso.

As discussões promovidas no espaço disponibilizado pelo ambiente virtual proporcionaram o enriquecimento das aulas presenciais. Realmente, pode-se observar que a ampliação dos espaços pedagógicos, com suporte da tecnologia, através das redes sociais online, podem ser um caminho de transposição dos limites impostos pela sala de aula. Além das reflexões realizadas, os estudantes também trouxeram soluções para problemas e desafios lançados pelo professor da disciplina.

Em muitos momentos, também ocorreram mediações entre o professor e os estudantes no ambiente online. Entretanto, o acompanhamento das contribuições e dúvidas de cada estudante foi dificultado pela forma linear e cronológica como as postagens são apresentadas. $\mathrm{O}$ docente, mediador da disciplina, deveria percorrer postagem por postagem para analisar as dúvidas e contribuições dos estudantes. Neste sentido, a ferramenta foi eficaz como um recurso de apoio pedagógico ao docente, visto que permitiu a organização das postagens por estudantes, bem como, a possibilidade de acompanhamento das necessidades de mediação individuais. Durante a execução do estudo piloto, os estudantes foram estimulados por meio de postagens do docente, as quais visaram provocar a participação em contribuições nas discussões acerca dos temas 
VII Congresso Brasileiro de Informática na Educação (CBIE 2018)

Anais do XXIX Simpósio Brasileiro de Informática na Educação (SBIE 2018)

abordados. Dessa forma, os estudantes puderam explorar um espaço além da sala de aula física, permitindo ampliar o tempo de discussão sobre temas abordados e, que muitas vezes, não tenham sido compreendidos.

Além disso, o estudo piloto demonstrou que o ambiente online favoreceu a reflexão e questionamentos por parte dos estudantes, motivando-os na formulação de perguntas, tentando resolver os problemas apresentados pelo docente, corroborando com as constatações de Souza e Moreira (2010), em seus estudos sobre os perfis de questionamento nos contextos de aprendizagem online. De acordo com os autores, as interações pedagógicas entre docente e estudante são complexas, sejam em sala de aula presencial, ou em ambientes virtuais, portanto, deve-se proporcionar condições para que o estudante seja o centro do processo de comunicação. Bautista et. al. (2006) complementam, informando que a comunicação multidirecional, proporcionada pela rede social, amplia a possibilidade de participação do estudante na discussão de temas relacionados a uma disciplina. Enquanto que, na comunicação unidirecional, presente na aula expositiva, o professor interage com os estudantes mas não há espaço para interação entre os próprios estudantes. Além disso, a comunicação proporcionada pelas redes sociais amplia os níveis de interação com os conteúdos postados pelos componentes do grupo.

Souza e Moreira (2010) destacam, ainda, que os ambientes online permitem o desenvolvimento da capacidade dos estudantes de formularem suas próprias perguntas, transformando o nível de interação pedagógica de meros expectadores para agentes ativos no desenvolvimento de sua própria aprendizagem. Este foi um aspecto relevante a ser ressaltado com o resultado da investigação, onde muitos estudantes trocaram informações entre si, num processo de construção mútua e colaborativa da aprendizagem. Notou-se o desenvolvimento da capacidade argumentativa em relação aos questionamentos do professor da disciplina. A elaboração de questões relacionadas aos temas trabalhados em sala de aula e discussão entre os próprios estudantes foi a tônica do espaço virtual.

Em relação as potencialidades pedagógicas da ferramenta Socialsearch, pode-se constatar sua utilidade na organização e agrupamento das postagens por estudantes, $\mathrm{o}$ que permitiu ao professor o acompanhamento do nível de participação individual, bem como, a observação de como ocorreram as interações entre os estudantes. Outro aspecto relevante a ser destacado foi o acompanhamento de estudantes pouco participativos. Notou-se que alguns estudantes que são participativos na sala de aula presencial não tiveram muito interesse nas discussões realizada no ambiente. Tal aspecto pode ser observado a partir dos cartões que sumarizam as interações de cada participante.

\section{Considerações finais}

A partir da análise dos resultados apontados pelo estudo piloto, apresentam-se algumas considerações a título de conclusão do trabalho investigativo. As conclusões aqui apresentadas estão limitadas as particularidades e limitações do estudo realizado, entretanto, permitem a identificação de elementos para interpretação de fenômenos no processo educativo, mais especificamente nas possibilidades de utilização da tecnologia no apoio a mediação pedagógica em ambientes online.

Com os resultados do trabalho, pode-se notar que a tecnologia pode ser um instrumento que proporciona condições para motivação do estudante durante o processo 
VII Congresso Brasileiro de Informática na Educação (CBIE 2018)

Anais do XXIX Simpósio Brasileiro de Informática na Educação (SBIE 2018)

educativo. Não pelo simples fato do professor adotar a tecnologia para replicação de materiais, mas como um meio de ampliar oportunidades para reflexão e discussão de temas complexos abordados nas disciplinas de um curso. As redes sociais, além de conectar pessoas, podem ser exploradas para fins educativos, desde que o professor as utilize como um recurso pedagógico, a partir de um planejamento prévio das atividades. $\mathrm{O}$ uso do ambiente virtual do Facebook como um instrumento para mediação pedagógica não é adequado sem algum artefato tecnológico de apoio, visto que as interações são postadas linearmente e de acordo com uma classificação cronológica. A tarefa de acompanhamento do desenvolvimento da aprendizagem do estudante é complexa nesta estrutura organizacional da rede social. Portanto, a Socialsearch demonstrou que é possível contornar-se tais limitações do Facebook, através de uma tecnologia complementar que extrai e organiza as interações entre o professor e estudante, bem como, as interações realizadas entre os próprios estudantes.

A primeira versão da ferramenta ainda é um protótipo, mesmo assim conseguiu cumprir o seu papel no mapeamento dos dados coletados do Facebook, porém ainda se têm um caminho a ser percorrido para o aperfeiçoamento da mesma e adições de novas funcionalidades e módulos, os quais trarão mais valor pedagógico através de informações mais detalhadas sobre os tipos de interações ocorridas no ambiente, visando-se auxiliar o professor mediador.

Um estudo sobre como ocorrem as interações de interesse pedagógico em um grupo de uma disciplina no Facebook seria um possível desdobramento do trabalho. $\mathrm{O}$ conteúdo textual das interações podem trazer pistas sobre como desenvolver um mecanismo para classificação automática do nível de mediação que se encontram os estudantes. A Classificação automática do nível de mediação dos estudantes visa amenizar a sobrecarga do professor durante o acompanhamento do desenvolvimento da aprendizagem dos estudantes com suporte de ambientes virtuais.

Outro desdobramento do trabalho seria a elaboração de um módulo de recomendação, onde a ferramenta poderia sugerir estratégicas pedagógicas ao professor da disciplina, a partir do mapeamento e análise das interações textuais no grupo do Facebook.

Por fim, evidenciou-se, a partir dos resultados da abordagem investigativa, que o ensino e aprendizagem não estão limitados às interações face a face, promovidas pela sala de aula presencial. Também é possível estender o espaço de mediação por meio de ambientes virtuais em uma forma de comunicação assíncrona.

\section{Referências bibliográficas}

Andrade, A. F.; Vicari, R. M. (2011) Construindo um ambiente de aprendizagem a distância inspirado na concepção sociointeracionista de Vygotsky. In SILVA, M. (Ed). Educação online: teorias, práticas, legislação, formação corporativa. $3^{\text {a }}$ Ed. São Paulo: Loyola, p.259-261.

Bautista, G.; Borges, F.; Forés, A. (2006) Didática Universitaria en Entornos Virtuales de Enseñanza Aprendizaje. Madrid: Narcea Ediciones.

Capra, F. (2008) Vivendo Redes. In DUARTE, F.; QUANDT, C.; SOUZA, Q. (Ed). O tempo das redes. São Paulo: Perspectiva, 2008. p.17-29.

Cord, B. (2018) Internet et pédagogie - état des lieux. Disponível em: $<\underline{\text { http://wwwadm.admp6.jussieu.fr/fp/uaginternetetp/definition travail collaboratif.ht }}$ 
VII Congresso Brasileiro de Informática na Educação (CBIE 2018)

Anais do XXIX Simpósio Brasileiro de Informática na Educação (SBIE 2018)

10

m> Acesso em: 01 mar. 2018.

Filatro, A.; Piconez, S. (2013) Evolução dos sistemas para educação a distância. In MACIEL, C. (Ed). Educação a Distância: Ambientes Virtuais de Aprendizagem. $1^{\text {a }}$ Ed. Cuiabá: EdUFMT, p.59-91.

Guedes-Granzotti, R. B.; Silva, K.; Dornelas, R.; Cphar, C.; Pellicani, A. D.; Domenis, D. R. (2015) Situação-problema como disparador do processo de ensinoaprendizagem em metodologias ativas de ensino. Revista CEFAC, n.17, v.6, p.20812087. Disponível em: <http://dx.doi.org/10.1590/1982-021620151768015>. Acesso em: 08 mar. 2018.

Harassim, L. (1989) Online education a new domain. In MASON, R.; KAYE, A. (Ed). Mindweave: comunications, computers and distance instruction. Oxford: Pergamon.

Juliani, D. P.; Juliani, J. P.; Souza, J. A.; Bettio, R. W. (2012) Utilização das redes sociais na educação: guia para o uso do Facebook em uma instituição de ensino superior. Revista Novas Tecnologias na Educação, Porto Alegre, n.10, v.3, p.1-11. Disponível em: <http://dx.doi.org/10.22456/1679-1916.36434>. Acesso em: 08 mar. 2018.

Moore, M. (2007) The Theory of Transactional Distance. In MOORE, M. G. (Ed). The Handbook of Distance Education. p.89-108, Mahwah, N.J. Lawrence Erlbaum Associates.

Moran, J. M. (2015) Mudando a educação com metodologias ativas. Convergências midiáticas, educação e cidadania: aproximações jovens, Ponta Grossa: UEPG Foca.

Oliveira, M. K. (2005) Vygotsky: aprendizado e desenvolvimento, um processo sócio-histórico. $4^{\mathrm{a}}$ ed. São Paulo: Scipione.

Paiva, M. R.; Parente, J. R.; Brandão, I. R.; Queiroz, A. H. (2016) Metodologias ativas de ensino-aprendizagem: revisão integrativa. Revista Sanare, n.15, v.2.

Panitz, T., Panitz, P. (1998) Ways To Encourage Collaborative Teaching In Higher Education. In JAMES, J. F. (Ed). University Teaching: International Perspectives, p.161-202. Garland Publishers: New York.

Prensky, M. H. (2009) Sapiens digital: from digital immigrants and digital natives to digital wisdom. Innovate: journal of online education, n.5, v.3, p.1-10.

Rangel, F. O.; De-Angelis, C.; Martins, R. L.; Costa, H. (2008) Mediação pretendida ou possível? Uma questão de tempo. Revista Intercâmbio, São Paulo: PUC-SP, n.17, v.1, p.177-192.

Silva, L. S.; Cotta, R. M. M.; Costa, G. D.; Campos, A. A. O. (2014) Formação de profissionais críticos e reflexivos: o potencial das metodologias ativas de ensinoaprendizagem e avaliação na aprendizagem significativa. Revista CIDUI, n.1, v.2, p.1-10.

Souza, F. N.; Moreira, A. (2010) Perfis de questionamento em contextos de aprendizagem online. Revista Iberoamericana de Informática Educativa, n.12, v.1, p.15-25.

Wellman, B. (1997) An Electronic Group is Virtually a Social Network. In KIESLER, S. (Ed), Culture of Internet. p.179-205, Hilsdale: Lawrence Erlbaum. 